\title{
Ein Mädchen mit juveniler idiopathischer Arthritis verliert an Gewicht - steckt da mehr dahinter?
}

Die juvenile idiopathische Arthritis (JIA) ist die häufigste chronisch entzündliche rheumatische Erkrankung des Kindesund Jugendalters und kann - insbesondere bei nicht ausreichend kontrollierter Aktivität - mit einer Beeinträchtigung von Wachstum und Gewicht einhergehen. Dennoch sollte auch an andere Ursachen gedacht werden, falls Längen- und/oder Gewichtsentwicklung Auffälligkeiten zeigen und zum sonstigen klinischen Befund, wie der Krankheitsaktivität der JIA, nicht passen.

\section{Kasuistik}

\section{Anamnese und klinische Unter- suchung}

Im Folgenden wird der Fall eines Mädchens vorgestellt, welches im Alter von 2 1/2 Jahren mit einer Bewegungseinschränkung des linken Kniegelenks auffiel. Das Kind wurde zunächst kinderorthopädisch vorgestellt: Klinisch fanden sich eine Schwellung des linken Kniegelenkes, eine schmerzhafte Bewegungseinschränkung mit einem Streckdefizit von 5 Grad sowie eine diskrete Quadrizepsatrophie. Das angefertigte Röntgenbild zeigte keinerlei Auffälligkeiten. Eine MRT in Sedierung gelang nicht, da es zu Bewegungsartefakten kam. In der diagnostischen Arthroskopie zeigte sich eine hypertrophe Synovialitis, im histologischen Befund wurde eine unspezifische Entzündung beschrieben. Daraufhin wurde das Mädchen in unsere kinderrheumatologische Sprechstunde überwiesen. In der Anamnese gab der Vater ein humpelndes Gangbild sowie eine Morgensteifigkeit von ca. 30 Minuten an. Bei der klinischen Untersuchung war das linke Kniegelenk geschwollen und überwärmt, es bestand ein Streckdefizit von $20^{\circ}$, die Beugung betrug maximal $90^{\circ}$; der übrige pädiatrisch-internistische und Gelenk-Status waren unauffällig; das Körpergewicht lag bei $12,0 \mathrm{~kg}$, die Körperlänge bei $89,5 \mathrm{~cm}$.

\section{Laborchemische Untersuchung}

Laborchemisch zeigten sich keine systemischen Entzündungszeichen; Serumionogramm, Leber- und Nierenwerte sowie Differenzial-Blutbild waren unauffällig. Bei normalen Erythrozytenzahlen von 5,5/pl war der Hämoglobin-Wert etwas erniedrigt (10,3 g/dl, Norm 10,8-14,3 g/ dl) bei auffällig hypochrom-mikrozytären Indizes (MCV $58 \mathrm{fl}$ [Referenz-Bereich 68-84 fl], MCH 18,9 pg [Referenz-Bereich 23-29 pg]), das Ferritin lag mit $80 \mu \mathrm{g} / \mathrm{l} \mathrm{im}$ Normbereich (20-200 $\mu \mathrm{g} / \mathrm{I})$ ebenso wie IgG, IgM und IgA. In der Autoimmundiagnostik fand sich ein ANA-Titer von 1:160 mit dicht feingranulärem nukleolärem Fluoreszenzmuster, der Rheumafaktor war negativ, das HLA-B27-Antigen positiv.

Mittels Ultraschall wurde nicht nur im linken, sondern auch im rechten Kniegelenk eine Aufweitung des Recessus suprapatellaris durch echoarme Flüssigkeit nachgewiesen; links auch mit Synovialisproliferationen.

\section{Diagnose, Therapie und Verlauf}

Es wurde die Diagnose einer juvenilen idiopathischen Arthritis mit oligoartikulärem Gelenkbefall gestellt und zunächst mit nichtsteroidalen Antirheumatika (NSAR; Ibuprofen, dann Indometacin) behandelt. Bei unzureichender klinischer Besserung wurden Punktionen beider Kniegelenke mit intraartikulärer Injektion von Triamcinolonhexacetonid durchgeführt. Im weiteren Verlauf zeigte sich eine vollständige Befundnormalisierung rechts, links persistierten die Synovialisproliferationen sowie ein geringer Erguss. Auch nach einer niedrig-dosierten kurzzeitigen systemischen Glukokortikoid-Therapie und physiotherapeutischen Behandlungen persistierten Morgensteifigkeit sowie Streck- als auch Beugedefizit im linken Kniegelenk.

Eine zunehmende Muskelatrophie und Wachstumsstörung des linken Beins mit resultierender Beinlängendifferenz führten dann zu dem Entschluss im Alter von 3 7/12 Jahren eine Basistherapie mit Methotrexat (MTX) zu beginnen. Die Gabe von NSAR wurde ein halbes Jahr später beendet. Unter dieser Therapie zeigte sich eine gute Kontrolle der Entzündungsaktivität im linken Kniegelenk mit im Verlauf ca. eines Jahres vollständiger Rückbildung von Erguss, Synovialisproliferationen, Bewegungseinschränkung und Beinlängendifferenz. Die augenärztlichen Kontrolluntersuchungen ergaben keinen Hinweis auf eine anteriore Uveitis.

Im Alter von 6 1 1 2 Jahren flammte kurzzeitig die Entzündung im linken Kniegelenk wieder etwas auf, vorübergehend wurden erneut zusätzlich NSAR verordnet. Im weiteren Verlauf wurde bei inaktiver Erkrankung das MTX zunächst reduziert und schließlich im Alter von 8 10/12 Jahren abgesetzt. Vier Monate später kam es dann zu einem Rezidiv der Grunderkrankung mit Arthritiden im linken Hüft-, rechten Knie- sowie rechten Kiefergelenk, sodass erneut eine Basistherapie mit MTX eingeleitet wurde, zusätzlich erhielt die Patientin NSAR und Physiotherapie. In den Laborkontrollen fanden sich weiterhin deutlich hypochrom-mikrozytäre Erythrozyten bei grenzwertig niedrigen Hämoglobin-Werten bei unauffälligem weißen Blutbild, negativen Entzündungszeichen sowie normwertigen Eisenstoffwechsel-Parametern. Diese Konstellation führte zur Durchführung einer Hämoglobin-Elektrophorese, die die Diagnose einer heterozygoten Alpha-Thalassämie (Alpha-Thalassämia minima) ergab, welche auch mittels genetischer Untersuchung verifiziert werden konnte.

Unter der MTX-Therapie wurde rasch erneut eine inaktive Erkrankung erreicht. Bei zunehmender subjektiver MTX-Intoleranz und andauernder Beschwerdefreiheit wurde MTX im Alter von knapp 12 Jahren wiederum abgesetzt. Bei den folgenden Routinekontrolluntersuchungen in der kinderrheumatologischen Sprechstunde fielen im Verlauf ein vermindertes Längenwachstum sowie eine Gewichtsabnahme auf ( Abb. 1), weiterhin zeigte das Mädchen noch keine Pubertätsentwicklung. Sowohl die rheumatologischen Untersuchungen als auch die allgemein-pädiatrische Anamnese waren zu dieser Zeit stets unauffällig. Es bestanden keine vermehrten Infekte, weder Bauchschmerzen noch Stuhlauffälligkeiten. In der Labordiagnostik im Alter von 13 Jahren fanden sich Transglutaminase (TG)-AK (TG-IgA $189 \mathrm{U} / \mathrm{ml}[$ Ref. < $10 \mathrm{U} / \mathrm{I}]$, TG-IgG $101 \mathrm{U} / \mathrm{ml}$ [Ref.<10 U/I]) sowie Endomysium-IgA-Antikörper. Die Bestimmung der Schilddrüsen-Autoantikörper zu diesem 


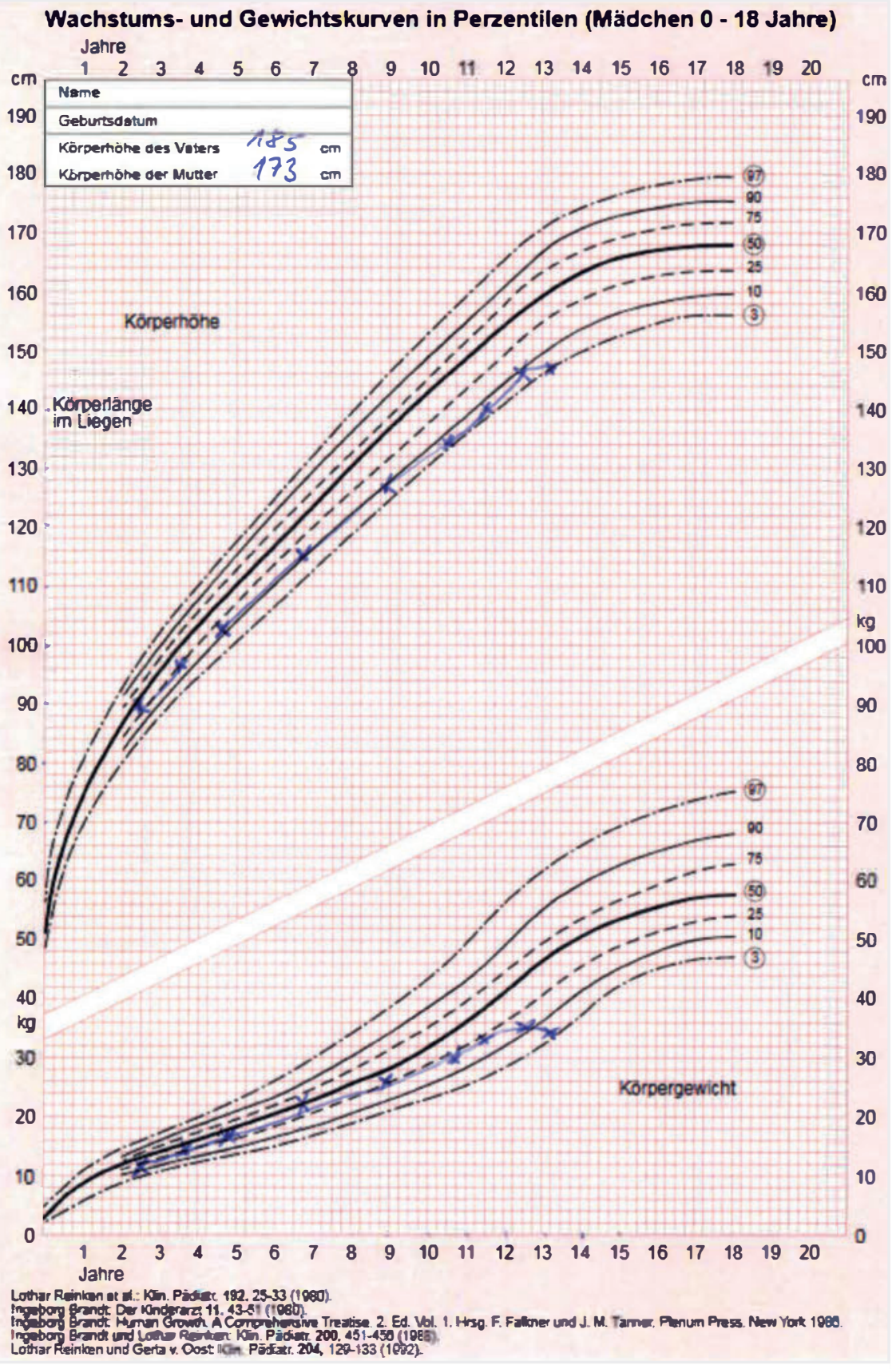

- Abb. 1 Vermindertes Längenwachstum und Gewichtsabnahme bei einem Mädchen mit juveniler idiopathischer Arthritis und einer zusätzlichen Zöliakie. (Quelle: Reinken und van Ost 1992)

Zeitpunkt war negativ; TSH, Trijodthyronin sowie Thyroxin waren im Normbereich.

\section{Diskussion}

Die Ursachen für ein verzögertes Wachstum, eine Gewichtsabnahme oder einen späten Eintritt in die Pubertät sind vielfältig. Eine chronisch-entzündliche Erkrankung, wie die JIA, kann bei über einen längeren Zeitraum persistierender Entzündungsaktivität zu einer Beeinträchtigung weitere Autoimmunerkrankung zu entwickeln, bei Patienten mit JIA größer als bei Gesunden.

Bei der Zöliakie unterscheidet man das sogenannte „klassische“ Krankheitsbild mit Durchfällen, distendiertem Abdomen und Malabsorptionsfolgen von den nicht klassischen Manifestationen, wie neurologische Symptome, Leberveränderungen mit Transaminasenerhöhungen, Anämie, Wachstumsretardierung, verzögerte Pubertätsentwicklung, aber auch Gelenkbeschwerden (zusammengefasst in [4]). Nicht alle diese klinischen Ausprägungen lassen sich als Folge der autoimmun vermittelten Enteropathie mit resultierender Atrophie der Darmschleimhaut und konsekutiver Malabsorption von Nährstoffen erklären. Mögliche pathophysiologische Zusammenhänge sind z. B. erhöhte Interleukin-Spiegel und Aktivierung von Immunzellen als Folgen der chronischen Entzündungsreaktion im Darm. Offenbar finden aber auch selbstständige autoimmune Prozesse in anderen Organen bzw. Geweben als dem Darm statt; so hat man z. B. TG-Antikörper gefunden, die mit Strukturen des Kleinhirns interagieren, oder auch Ablagerungen von TG-Antikörpern in der Leber von Zöliakie-Patienten. Sogar eine Interaktion mit den Follikeln der Schilddrüse ist beschrieben worden, die möglicherweise unabhängig von der Ausbildung spezifischer anti-Thyreoglobulin-Ak oder anti-Thyreoperoxidase-AK zu einer Schilddrüsenfunktionsstörung führt [5].

Aus dem klinischen Verlauf, der Wachstums- und Gewichtsentwicklung lässt sich ableiten, dass unsere Patientin zuerst an einer JIA erkrankte und dann sekundär - als autoimmunologische Zweiterkrankung - eine Zöliakie entwickelte. Die Schilddrüsen (SD)-Parameter sowie die spezifischen SD-Autoantikörper wurden untersucht, da erstens das Risiko für eine Autoimmunthyreoiditis bei bereits bestehender Autoimmunerkrankung erhöht ist und zweitens die Schilddrüsenfunktion bei Vorliegen einer Wachstumsverzögerung untersucht werden sollte. Die Arthritis war über einen langen Zeitraum vor Diagnosestellung der Zöliakie inaktiv, die Medikation abgesetzt, sodass die JIA nicht als ursächlich für das verminderte Längenwachstum und den Gewichtsrückgang angesehen werden 
konnte. Auch wenn sie keine „klassischen“ Beschwerden wie Meteorismus und Diarrhöen angab, ist die Patientin aufgrund ihrer verzögerten somatischen Entwicklung als symptomatisch hinsichtlich einer Zöliakie einzuordnen.

Die ESPGHAN gibt in ihrer klinischen Leitlinie Empfehlungen, bei welchen Patienten eine Diagnostik bezüglich Zöliakie durchgeführt werden sollte [6]. In der Gruppe 1 werden Kinder und Jugendliche mit folgenden Auffälligkeiten zusammengefasst: chronische oder intermittierende Diarrhöen, Gewichtsverlust, beeinträchtigtes Wachstum oder eine verzögerte Pubertätsentwicklung, Amenorrhö, Eisenmangelanämie, Übelkeit oder Erbrechen, chronische Bauchschmerzen, Bauchkrämpfe oder abdominelle Distension, chronische Verstopfung, chronische Müdigkeit, wiederkehrende orale Aphthen, Dermatitis-herpetiformis-artiger Ausschlag, Hinweise auf eine Osteoporose/Osteopenie, z. B. bei Fraktur nach inadäquatem Trauma sowie abnorme Leberwerte. Bei derartigen Symptomen/ Befunden sollte entsprechend der Leitlinie ein Test auf anti-Transglutaminase-IgA-AK (Voraussetzung: kein IgA-Mangel, ansonsten sollen anti-TG-IgG bestimmt werden) erfolgen. Ist dieser positiv, sollte zunächst ein 2. Test auf Zöliakie-typische Antikörper folgen, in der Regel auf anti-Endomysium-Antikörper (EMA). Ist dieser ebenfalls positiv, sollte die Diagnose einer Zöliakie durch die histologische Untersuchung einer duodenalen Biopsie bestätigt werden. Auf diese kann nach der zitierten Leitlinie verzichtet werden, wenn der anti-TGAK-Serumwert mehr als 10 fach über dem Grenzwert liegt - so wie im Fall der vorgestellten Patientin mit anti-TG-AK von 189U/ $\mathrm{ml}$ bei einem Referenzbereich $<10 \mathrm{U} / \mathrm{ml}$.

Zusätzlich kann eine HLA-Typisierung hilfreich sein: Vorliegen von HLA-DQ2 und -DQ8 stützt die Diagnose einer Zöliakie, während kein Nachweis dieser HLA-Allele die Diagnose unwahrscheinlich macht und daher andere Differenzialdiagnosen für die entsprechende Klinik in Betracht gezogen werden sollten. Hier, sowie in den Fällen, bei denen die Zöliakie-typischen Antikörper (anti-TG-AK, EMA, Antikörper gegen deamidierte Gliadin-Peptide [anti-GDP]) in geringerer Menge vorliegen, ist die Biopsie der Duodenalschleimhaut mit histolo-

- Tab. 1 Studien zur Häufigkeit einer Zöliakie bei Patienten mit einer juvenilen idiopathischen Arthritis; nach [7-12]

\section{Studien mit Patienten mit JIA}

Lepore 1996, $n=119$

George 1996, n=62

Stagi $2005, n=151$

Alpigiani 2008, n= 107

Pohjankoski 2010, $\mathrm{n}=417$

Robazzi 2013, n= 53

\section{Zusätzlich Diagnose Zöliakie (bestätigt durch Biopsie)}

$2,5 \%$

$1,5 \%$

6,7\% (Kontrollgruppe 0,6\%)

2,8\% (nach einem FU von 5,5 Jahren [Median])

$0,7 \%$

$2 \%$ gischer Aufarbeitung die zielführende Methode.

Es gibt ein paar Arbeiten, die das Auftreten einer Zöliakie bei Patienten mit JIA und umgekehrt beschrieben haben ( $>$ Tab. 1). Diese unterscheiden sich hinsichtlich mehrerer Parameter, wie z. B. Patientenzahl, Krankheitsdauer, Follow-up der Patienten, sodass es nicht erstaunt, dass recht unterschiedliche Prävalenzen der Zöliakie in den JIA-Kollektiven beschrieben werden. Einige Patienten mit JIA und Zöliakie wiesen zudem noch eine Autoimmunthyreoiditis auf. So wurde in der Studie von Stagi et al. [9] bei 11,9\% eine solche bei JIA-Patienten diagnostiziert (Vorliegen von SDAutoantikörpern und erhöhten TSH-Werten und/oder typischer Hypoechogenität in der Sonografie der Schilddrüse), in der Kontrollgruppe dagegen nur bei 1,2\%. Ein ähnliches Ergebnis beschrieben auch Robazzi et al. [12] in einer Untersuchung von 53 JIA-Patienten, von denen $11 \%$ auch eine Autoimmunthyreoiditis hatten, während in der Kontrollgruppe keiner diese Diagnose bekam.

Die Prävalenz der Zöliakie bei Kindern und Jugendlichen unter 18 Jahren wurde im Rahmen der Studie zur Gesundheit von Kindern und Jugendlichen in Deutschland (KIGGS) untersucht und mit ca. 0,9\% ermittelt [13].

Aufgrund des höheren Risikos für weitere Autoimmunerkrankungen stellt sich für $\mathrm{Pa}$ tienten mit einer JIA oder anderen Autoimmunerkrankung die Frage des Screenings auf weitere, möglicherweise asymptomatische Autoimmunerkrankungen, wie eine Zöliakie. Die ESPGHAN empfiehlt in ihrer Leitlinie eine Testung auf Zöliakie-spezifi- sche Antikörper bei asymptomatischen Kindern und Jugendlichen, die aufgrund der nachfolgend aufgeführten Erkrankungen ein erhöhtes Risiko für das Auftreten einer Zöliakie haben (Gruppe 2, in Klammern jeweils die Prävalenz von Zöliakie bei dieser Erkrankung, zusammengefasst in [12]):

- Diabetes mellitus Typ 1 (4,5-13,5\%),

- Down-Syndrom (3,0-13,0\%),

- Autoimmunthyreoiditis (1,3-6,2\%),

- Turner-Syndrom (9,4-12,5\%),

- Williams-Beuren-Syndrom (4,2\%),

- selektiver IgA-Mangel, Autoimmunhepatitis;

- außerdem, falls Verwandte ersten Grades unter Zöliakie leiden (3,1-8,9\%).

Hier sollte zunächst eine Untersuchung auf das Vorliegen von HLA-DQ2 und -DQ8 erfolgen, da bei negativem Befund die Diagnose Zöliakie unwahrscheinlich ist und auch keine Bestimmung spezifischer Antikörper mehr erfolgen sollte. Bei positivem Ergebnis sollte sich die Untersuchung auf antiTG-AK anschließen, welche bei wiederum positivem Befund von der Untersuchung auf EMA als Bestätigungstest gefolgt sein sollte. Wegen der weitreichenden Konsequenzen der Diagnose Zöliakie mit lebenslanger (!) und nicht immer ganz einfach umzusetzender Diät empfiehlt die ESPGHAN zur Diagnosesicherung bei asymptomatischen Kindern und Jugendlichen der Gruppe 2 die duodenale Biopsie mit histologischem Nachweis einer Enteropathie.

Aus den hier vorgestellten Arbeiten geht hervor, dass die juvenile idiopathische Arthritis zwar ein erhöhtes Risiko für die Entwicklung einer Zöliakie mit sich bringt, die angegebenen Prävalenzraten aber meistens unter denen für die oben aufgeführten „Risikoerkrankungen“ liegen (mean 
2,7\%); sicherlich deshalb wird die JIA in der ESPGHAN-Leitlinie auch nicht aufgeführt. Daher erscheint ein generelles Screening von JIA-Patienten auf Zöliakie zum jetzigen Zeitpunkt nicht angezeigt; möglicherweise werden neue Erkenntnisse zu molekulargenetischen und immunologischen Zusammenhängen diese Einschätzung revidieren.

\section{Interessenkonflikt}

CS: keine

KM: Vortragshonorare von AbbVie, Biermann, Chugai, Medac, Roche

\section{Einhaltung ethischer Richtlinien}

Das Einverständnis der Mutter der Patientin zur Veröffentlichung der Kasuistik liegt vor.

\section{Autorinnen}

Claudia Sengler, Kirsten Minden Deutsches Rheuma-Forschungszentrum Berlin, Programmbereich Epidemiologie

\section{Korrespondenzadresse}

\section{Dr. Claudia Sengler}

Deutsches Rheuma-Forschungszentrum Berlin

Ein Institut der Leibnizgemeinschaft

Programmbereich Epidemiologie

Charitéplatz 1, 10117 Berlin

sengler@drfz.de
[1] Viken MK, Amundsen SS, Kvien TK et al. Association analysis of the 1858 C > T polymorphism in the PTPN22 gene in juvenile idiopathic arthritis and other autoimmune diseases. Genes Immun 2005; 6(3): 271-273

[2] Li Y, Cheng $\mathrm{H}$, Zuo XB et al. Association analyses identifying two common susceptibility loci shared by psoriasis and systemic lupus erythematosus in the Chinese Han population. J Med Genet 2013; 50(12): 812-818. doi: $10.1136 /$ jmedgenet-2013-101787. Epub 2013 Sep 26

[3] Kara S, Pirela-Morillo GA, Gilliam CT, Wilson GD. Identification of novel susceptibility genes associated with seven autoimmune disorders using whole genome molecular interaction networks. J Autoimmun 2019; 97: 48-58

[4] Laurikka P, Nurminen S, Kivelä L, Kurppa K. Extraintestinal Manifestations of Celiac Disease: Early Detection for Better Long-Term Outcomes. Nutrients 2018; 10(8)

[5] Naiyer AJ, Shah J, Hernandez L et al. Tissue transglutaminase antibodies in individuals with celiac disease bind to thyroid follicles and extracellular matrix and may contribute to thyroid dysfunction. Thyroid 2008; 18(11): 1171-1178

[6] Husby S, Koletzko S, Korponay-Szabó IR et al.; ESPGHAN Working Group on Coeliac Disease Diagnosis; ESPGHAN Gastroenterology Committee; European Society for Pediatric Gastroenterology, Hepatology, and Nutrition. European Society for Pediatric Gastroenterology, Hepatology, and Nutrition guidelines for the diagnosis of coeliac disease. J Pediatr Gastroenterol Nutr 2012; 54(1): 136-160

[7] Lepore L, Martelossi S, Pennesi M et al. Prevalence of celiac disease in patients with juvenile chronic arthritis. J Pediatr 1996; 129(2): 311-313
[8] George EK, Hertzberger-ten Cate R, van Suijlekom-Smit LW et al. Juvenile chronic arthritis and coeliac disease in The Netherlands. Clin Exp Rheumatol 1996; 14(5): 571-575

[9] Stagi S, Giani T, Simonini G, Falcini F. Thyroid function, autoimmune thyroiditis and coeliac disease in juvenile idiopathic arthritis. Rheumatology (Oxford) 2005; 44(4): 517-520

[10] Alpigiani MG, Haupt R, Parodi S et al. Coeliac disease in 108 patients with juvenile idiopathic arthritis: a 13-year follow-up study. Clin Exp Rheumatol 2008; 26(1): 162

[11] Pohjankoski H, Kautiainen $\mathrm{H}$, Kotaniemi $\mathrm{K}$ et al. Autoimmune diseases in children with juvenile idiopathic arthritis. Scand J Rheumatol 2010; 39(5): 435-436

[12] Robazzi TC, Adan LF, Pimentel K et al. Autoimmune endocrine disorders and coeliac disease in children and adolescents with juvenile idiopathic arthritis and rheumatic fever. Clin Exp Rheumatol 2013; 31(2): 310-317

[13] Laass MW, Schmitz R, Uhlig $\mathrm{HH}$ et al. The prevalence of celiac disease in children and adolescents in Germany. Dtsch Arztebl Int 2015; 112(33-34): 553-560

\section{Bibliografie}

DOI https://doi.org/10.1055/a-0868-5900 arthritis + rheuma 2019; 39: 126-129 (c) Georg Thieme Verlag KG Stuttgart · New York ISSN 0176-5167 